\title{
On the Brodutch and Modi method of constructing geometric measures of classical and quantum correlations
}

\author{
Zbigniew Walczak ${ }^{1}$ - Iwona Wintrowicz ${ }^{1}$
}

Received: 12 November 2015 / Accepted: 28 January 2017 / Published online: 11 February 2017

C The Author(s) 2017. This article is published with open access at Springerlink.com

\begin{abstract}
Recently, Brodutch and Modi proposed a general method of constructing meaningful measures of classical and quantum correlations. We systematically apply this method to obtain geometric classical and quantum correlations based on the Bures and the trace distances for two-qubit Bell diagonal states. Moreover, we argue that in general the Brodutch and Modi method may provide non-unique results, and we show how to modify this method to avoid this issue.
\end{abstract}

Keywords Quantum correlations · Classical correlations · Geometric measures of correlations $\cdot$ Bell diagonal states

\section{Introduction}

In quantum information science, the problem of classification and quantification of correlations present in quantum states has been widely studied in the last two decades $[1,2]$. In this regard, the most significant progress has been made in the case of bipartite quantum systems which have been studied initially in the entanglement-separability paradigm that was first formalized by Werner [3]. Within this paradigm, the correlations present in a quantum state can be classified as either classical or quantum, where the latter ones are identified with entanglement that can be quantified by a variety of entanglement measures [1].

\footnotetext{
$凶$ Zbigniew Walczak

z.walczak@merlin.phys.uni.lodz.pl

Iwona Wintrowicz

i.wintrowicz@merlin.phys.uni.lodz.pl

1 Department of Theoretical Physics, University of Lodz, Pomorska, 149/153, 90-236 Lodz, Poland
} 
However, it has gradually become clear that entanglement cannot be regarded as the only kind of quantum correlations, because separable quantum states can also have quantum correlations, other than entanglement, that are responsible for the improvements of some quantum tasks that cannot be simulated by classical methods [4-10]. Therefore, it has become evident that the entanglement-separability paradigm should be replaced by a new one.

The first step in this direction was taken independently by Ollivier and Zurek who introduced quantum discord as an information-theoretic measure of quantum correlations beyond entanglement [11] and by Henderson and Vedral who studied the problem of separation of classical and quantum correlations from an information-theoretic perspective [12]. Due to the discovery [13] that quantum discord may be the key resource in the deterministic quantum computation with one qubit [4], the problem of classification and quantification of correlations present in quantum states has been extensively studied within the information-theoretic paradigm [2].

Because quantum discord cannot be computed analytically even for arbitrary two-qubit states [2], an alternative approach to classification and quantification of correlations within the information-theoretic paradigm has been proposed in which different types of correlations are quantified by a distance from a given quantum state to the closest state which does not have the desired property [14]. Of course, within this approach the amount of quantum correlations present in a given quantum state is determined by the choice of distance measure for quantum states.

The first geometric measure of quantum correlations was geometric quantum discord in which the Schatten 2-norm has been applied as the distance measure between a given quantum state and the closest zero discord state to obtain the analytical expression for geometric quantum discord for a general two-qubit state [15]. Of course, geometric quantum discord has attracted considerable interest due to its analytic computability for general two-qubit states [2].

However, recently it has been shown that geometric quantum discord cannot be regarded as a bona fide measure of quantum correlations [16], because of the lack of contractivity of the Schatten 2-norm under trace-preserving quantum channels [17]. Moreover, it has turned out that among all geometric quantum discords based on the Schatten $p$-norms [18] only geometric quantum discord based on the Schatten 1-norm is a meaningful measure of quantum correlations [17].

The problems with geometric quantum discord have highlighted the need for a general method of constructing meaningful measures of correlations within the information-theoretic paradigm.

Recently, Brodutch and Modi [19] proposed a method in which quantum correlations are quantified by a distance between a given multipartite quantum state and the classical-quantum state emerging from a measurement performed on the considered state, where the measurement is chosen according to some strategy. Moreover, within this method classical correlations are quantified by a distance between the classical-quantum state and the completely separable state resulting from the same measurement performed on the tensor product of the states of the individual subsystems. Furthermore, Brodutch and Modi [19] identified the strategies that provide meaningful measures of classical and quantum correlations that satisfy the following necessary conditions: product states have no correlations, all correlations are invariant 
under local unitary operations, all correlations are non-negative, and classical states have no quantum correlations.

The purpose of this paper is twofold. First, we systematically apply the Brodutch and Modi method to obtain for the first time geometric classical and quantum correlations based on the Bures distance for two-qubit Bell diagonal states using two natural strategies for constructing meaningful measures of correlations. Second, we consider geometric classical and quantum correlations based on the trace distance for two-qubit Bell diagonal states to show that the Brodutch and Modi method should be modified as in this case one of two possible strategies results in the non-uniqueness of geometric classical correlations. Moreover, we show how to modify the Brodutch and Modi method to avoid the problem of non-unique results in the general case.

\section{Geometric classical and quantum correlations based on the Bures distance}

In this section, we systematically apply the Brodutch and Modi method to obtain geometric classical and quantum correlations based on the Bures distance for twoqubit Bell diagonal states using two natural strategies for constructing meaningful measures of correlations.

In the framework of the Brodutch and Modi method [19], quantum and classical correlations present in a multipartite state $\rho$ are quantified, respectively, by

$$
\begin{aligned}
& Q(\rho)=K[\rho, M(\rho)], \\
& C(\rho)=K\left[M(\rho), M\left(\pi_{\rho}\right)\right],
\end{aligned}
$$

where $K[\rho, \tau]$ is a non-negative real-valued function of states $\rho$ and $\tau$ that vanishes for $\tau=\rho, M$ is a measurement chosen according to some strategy, $M(\rho)$ is the classical-quantum state emerging from a measurement $M$ performed on $\rho, M\left(\pi_{\rho}\right)$ is the completely separable state resulting from the same measurement performed on $\pi_{\rho}$, and $\pi_{\rho}$ is the tensor product of the states of the individual subsystems. Moreover, within this method there are two natural strategies of choosing a measurement $M$ that provide meaningful measures of quantum and classical correlations present in a multipartite state $\rho$ [19]

- $M$ is a non-selective rank-1 projective measurement performed on one subsystem of the multipartite system in a state $\rho$, and $M$ minimizes the quantum correlations $Q(\rho)$,

- $M$ is a non-selective rank-1 projective measurement performed on one subsystem of the multipartite system in a state $\rho$, and $M$ maximizes the classical correlations $C(\rho)$.

One can apply the Brodutch and Modi method to define geometric quantum and classical correlations present in a multipartite state $\rho$ by adopting the Bures distance between states $\rho$ and $\tau$ as a function $K[\rho, \tau]$ 


$$
\begin{aligned}
& Q_{B}(\rho)=d_{B}(\rho, M(\rho)), \\
& C_{B}(\rho)=d_{B}\left(M(\rho), M\left(\pi_{\rho}\right)\right),
\end{aligned}
$$

with $d_{B}(\rho, \tau)=\sqrt{2-2 \sqrt{F(\rho, \tau)}}$ where $F(\rho, \tau)=(\operatorname{Tr}[\sqrt{\sqrt{\rho} \tau \sqrt{\rho}}])^{2}$ is the Uhlmann fidelity between states $\rho$ and $\tau$, and the measurement $M$ is chosen according to one of the above strategies.

A two-qubit Bell diagonal state can be written in the following form [20]

$$
\rho=\frac{1}{4}\left(I \otimes I+\sum_{m=1}^{3} c_{m} \sigma_{m} \otimes \sigma_{m}\right),
$$

where $I$ is the identity matrix, $\sigma_{m}$ are the Pauli matrices and real numbers $c_{m}$ fulfill the following conditions

$$
\begin{aligned}
& 0 \leq \frac{1}{4}\left(1-c_{1}-c_{2}-c_{3}\right) \leq 1, \\
& 0 \leq \frac{1}{4}\left(1-c_{1}+c_{2}+c_{3}\right) \leq 1, \\
& 0 \leq \frac{1}{4}\left(1+c_{1}-c_{2}+c_{3}\right) \leq 1, \\
& 0 \leq \frac{1}{4}\left(1+c_{1}+c_{2}-c_{3}\right) \leq 1 .
\end{aligned}
$$

The above inequalities describe a tetrahedron with vertices $(1,1,-1),(-1,-1,-1)$, $(1,-1,1)$, and $(-1,1,1)$ representing the Bell states [20]. Thus, there is a one-toone correspondence between two-qubit Bell diagonal states and points within the tetrahedron.

Let us note that if the measurement $M$, described by a complete set of onedimensional orthogonal projectors $\left\{\Pi_{ \pm}\right\}$, is performed on the first qubit of the two-qubit system in a Bell diagonal state, then the classical-quantum state $M(\rho)$ and the completely separable state $M\left(\pi_{\rho}\right)$ have the form

$$
\begin{aligned}
M(\rho) & =\sum_{m=+,-}\left(\Pi_{m} \otimes I\right) \rho\left(\Pi_{m} \otimes I\right), \\
M\left(\pi_{\rho}\right) & =\frac{1}{4}(I \otimes I),
\end{aligned}
$$

where $\Pi_{ \pm}=\frac{1}{2}(I \pm \mathbf{n} \cdot \boldsymbol{\sigma}), \mathbf{n}=\left(n_{1}, n_{2}, n_{3}\right)$ is a real three-dimensional unit vector and $\sigma=\left(\sigma_{1}, \sigma_{2}, \sigma_{3}\right)$.

To determine geometric classical and quantum correlations based on the Bures distance for two-qubit Bell diagonal states, we first need to find the Uhlmann fidelity between states $\rho$ and $M(\rho)$ and then the Uhlmann fidelity between states $M(\rho)$ and $M\left(\pi_{\rho}\right)$. One can show that in the case of two-qubit Bell diagonal states and the measurement $M$ described by $\left\{\Pi_{ \pm}\right\}$, the Uhlmann fidelity between states $\rho$ and $M(\rho)$ is given by 


$$
F(\rho, M(\rho))=\left(\sum_{i=1}^{4} \sqrt{\lambda_{i}}\right)^{2}
$$

where coefficients $\lambda_{i}$, being the eigenvalues of operator $A=\sqrt{\rho} M(\rho) \sqrt{\rho}$, are the solutions of the characteristic equation

$$
\lambda^{4}+\alpha_{3} \lambda^{3}+\alpha_{2} \lambda^{2}+\alpha_{1} \lambda+\alpha_{0}=0
$$

with

$$
\begin{aligned}
& \alpha_{3}=-\operatorname{Tr}(A), \\
& \alpha_{2}=-\frac{1}{2} \operatorname{Tr}\left(A^{2}\right)+\frac{1}{2}(\operatorname{Tr}(A))^{2}, \\
& \alpha_{1}=-\frac{1}{3} \operatorname{Tr}\left(A^{3}\right)+\frac{1}{2} \operatorname{Tr}(A) \operatorname{Tr}\left(A^{2}\right)-\frac{1}{6}(\operatorname{Tr}(A))^{3}, \\
& \alpha_{0}=\operatorname{Det}(A) .
\end{aligned}
$$

Although the coefficients $\lambda_{i}$ can be computed analytically, we do not report them here as the explicit expressions are too cumbersome. One can also show that in the case of two-qubit Bell diagonal states and the measurement $M$ described by $\left\{\Pi_{ \pm}\right\}$, the Uhlmann fidelity between states $M(\rho)$ and $M\left(\pi_{\rho}\right)$ is given by

$$
F\left(M(\rho), M\left(\pi_{\rho}\right)\right)=\frac{1}{2}\left(1+\sqrt{1-c_{1}^{2} n_{1}^{2}-c_{2}^{2} n_{2}^{2}-c_{3}^{2} n_{3}^{2}}\right) .
$$

The analytic expressions for $F\left(M(\rho), M\left(\pi_{\rho}\right)\right)$ and $F(\rho, M(\rho))$ make it possible to obtain geometric classical and quantum correlations based on the Bures distance for two-qubit Bell diagonal states under the two natural strategies of choosing the measurement $M$.

\subsection{Strategy 1}

In the framework of the first strategy, for a given two-qubit Bell diagonal state (5) we first identify the measurements $M$ that minimize the geometric quantum correlations (3) and then we use these optimal measurements to compute the geometric classical correlations (4). In other words, for a given point $\left(c_{1}, c_{2}, c_{3}\right)$ of tetrahedron (6) we first identify unit vectors $\left(n_{1}, n_{2}, n_{3}\right)$ that maximize the fidelity (9) and then we use these optimal vectors to compute the fidelity (12).

It can be shown that under this strategy

- if $c_{i}^{2}=c_{j}^{2}=c_{k}^{2}$ where $i \neq j \neq k$, then all measurements $M$ are optimal, and geometric quantum and classical correlations are given by 


$$
\begin{aligned}
& Q_{B}(\rho)=\sqrt{2-2 \sqrt{w_{k}}}, \\
& C_{B}(\rho)=\sqrt{2-\sqrt{2+2 \sqrt{1-c_{k}^{2}}}},
\end{aligned}
$$

- if $c_{i}^{2}=c_{j}^{2}<c_{k}^{2}$ where $i \neq j \neq k$, then only measurements $M$ with $n_{i}^{2}=n_{j}^{2}=0$ and $n_{k}^{2}=1$ are optimal, and geometric quantum and classical correlations are given by Eqs. (13),

- if $c_{i}^{2}<c_{j}^{2}=c_{k}^{2}$ where $i \neq j \neq k$, then only measurements $M$ with $n_{i}^{2}=0$ and $n_{j}^{2}+n_{k}^{2}=1$ are optimal, and geometric quantum and classical correlations are given by Eqs. (13),

- if $c_{i}^{2}<c_{j}^{2}<c_{k}^{2}$ where $i \neq j \neq k$, then only measurements $M$ with $n_{i}^{2}=n_{j}^{2}=0$ and $n_{k}^{2}=1$ are optimal, and geometric quantum and classical correlations are given by Eqs. (13),

where coefficients $w_{1}, w_{2}$ and $w_{3}$ have the following form

$$
\begin{aligned}
w_{1}= & \frac{1}{16}\left(\sqrt{\left(1-c_{1}\right)\left(1-c_{1}-c_{2}-c_{3}\right)}+\sqrt{\left(1-c_{1}\right)\left(1-c_{1}+c_{2}+c_{3}\right)}\right. \\
& \left.+\sqrt{\left(1+c_{1}\right)\left(1+c_{1}-c_{2}+c_{3}\right)}+\sqrt{\left(1+c_{1}\right)\left(1+c_{1}+c_{2}-c_{3}\right)}\right)^{2}, \\
w_{2}= & \frac{1}{16}\left(\sqrt{\left(1-c_{2}\right)\left(1-c_{1}-c_{2}-c_{3}\right)}+\sqrt{\left(1+c_{2}\right)\left(1-c_{1}+c_{2}+c_{3}\right)}\right. \\
& \left.+\sqrt{\left(1-c_{2}\right)\left(1+c_{1}-c_{2}+c_{3}\right)}+\sqrt{\left(1+c_{2}\right)\left(1+c_{1}+c_{2}-c_{3}\right)}\right)^{2}, \\
w_{3}= & \frac{1}{16}\left(\sqrt{\left(1-c_{3}\right)\left(1-c_{1}-c_{2}-c_{3}\right)}+\sqrt{\left(1+c_{3}\right)\left(1-c_{1}+c_{2}+c_{3}\right)}\right. \\
& \left.+\sqrt{\left(1+c_{3}\right)\left(1+c_{1}-c_{2}+c_{3}\right)}+\sqrt{\left(1-c_{3}\right)\left(1+c_{1}+c_{2}-c_{3}\right)}\right)^{2} .
\end{aligned}
$$

The above results show that geometric classical and quantum correlations based on the Bures distance for two-qubit Bell diagonal states are uniquely determined under the first strategy, despite the fact that for a wide class of two-qubit Bell diagonal states we have more than one optimal measurement $M$ which means that for those states the classical-quantum state $M(\rho)$ cannot be uniquely determined. Therefore, in general non-uniqueness of $M(\rho)$ does not necessarily imply non-uniqueness of geometric classical correlations under the first strategy. In the next section, we show that non-uniqueness of $M(\rho)$ may, however, imply non-uniqueness of geometric classical correlations under this strategy.

Let us note here that the problem of identification of unit vectors $\left(n_{1}, n_{2}, n_{3}\right)$ maximizing the fidelity (9) for a given point $\left(c_{1}, c_{2}, c_{3}\right)$ of tetrahedron (6) is closely related to the problem of finding classical-quantum states $\chi_{\rho}$ that maximize the fidelity $F\left(\rho, \chi_{\rho}\right)$. This problem has been studied in the literature [21,22] in the context of the Bures geometric quantum discord in which the Bures distance was applied as the distance measure between a given quantum state and the closest classical-quantum state. Interestingly, in general the Bures geometric quantum discord $D_{B}(\rho)$ [23] is less than 
or equal to geometric quantum correlations based on the Bures distance under the first strategy $Q_{B}(\rho)$, i.e., $D_{B}(\rho) \leq Q_{B}(\rho)$, since $M(\rho)$ is always a classical-quantum state for a bipartite state $\rho$ [24]. Of course, the relation $D_{B}(\rho) \leq Q_{B}(\rho)$ holds in the case of two-qubit Bell diagonal states as one can verify taking into account our results regarding $Q_{B}(\rho)$ and those found in the literature regarding $D_{B}(\rho)$ [23]. Moreover, one can show that this inequality becomes an equality if and only if $\rho=M(\rho)$ for optimal measurement $M$ or $\rho$ is a mixture of two Bell states. It is also worth noting that considering the Bures geometric quantum discord for two-qubit Bell diagonal states one cannot uniquely determine the closest classical-quantum state for a wide class of two-qubit Bell diagonal states [22]. More precisely, it can be done uniquely if and only if a two-qubit Bell diagonal state is represented by the point $\left(c_{1}, c_{2}, c_{3}\right)$ being interior point of tetrahedron (6) and the index $k$ such that $c_{k}^{2}=\max \left(c_{1}^{2}, c_{2}^{2}, c_{3}^{2}\right)$ is uniquely given. For comparison, in the case of geometric quantum correlations based on the Bures distance under the first strategy the classical-quantum state $M(\rho)$ can be uniquely determined if and only if a two-qubit Bell diagonal state is represented by the point $\left(c_{1}, c_{2}, c_{3}\right)$ of tetrahedron (6) and the index $k$ such that $c_{k}^{2}=\max \left(c_{1}^{2}, c_{2}^{2}, c_{3}^{2}\right)$ is uniquely given, as it was shown above. Moreover, it is worth noting that an alternative approach to geometric classical correlations based on the Bures distance under the first strategy has been considered in the literature [23]. The classical correlations measure based on the Bures distance introduced in [23] can be computed analytically for two-qubit Bell diagonal states, like geometric classical correlations based on the Bures distance under the first strategy. However, it can be shown that they are not directly comparable measures of classical correlations, because unlike for $D_{B}(\rho)$ and $Q_{B}(\rho)$ for which the relation $D_{B}(\rho) \leq Q_{B}(\rho)$ holds, a similar relation, valid for all two-qubit Bell diagonal states, cannot be established between these two measures of classical correlations.

\subsection{Strategy 2}

In the framework of the second strategy, for a given two-qubit Bell diagonal state (5) we first identify the measurements $M$ that maximize the geometric classical correlations (4) and then we use these optimal measurements to compute the geometric quantum correlations (3). In other words, for a given point $\left(c_{1}, c_{2}, c_{3}\right)$ of tetrahedron (6) we first identify unit vectors $\left(n_{1}, n_{2}, n_{3}\right)$ that minimize the fidelity (12) and then we use these optimal vectors to compute the fidelity (9).

It can be shown that under this strategy

- if $c_{i}^{2}=c_{j}^{2}=c_{k}^{2}$ where $i \neq j \neq k$, then all measurements $M$ are optimal, and geometric quantum and classical correlations are given by

$$
\begin{aligned}
& Q_{B}(\rho)=\sqrt{2-2 \sqrt{w_{k}}}, \\
& C_{B}(\rho)=\sqrt{2-\sqrt{2+2 \sqrt{1-c_{k}^{2}}}},
\end{aligned}
$$


- if $c_{i}^{2}=c_{j}^{2}<c_{k}^{2}$ where $i \neq j \neq k$, then only measurements $M$ with $n_{i}^{2}=n_{j}^{2}=0$ and $n_{k}^{2}=1$ are optimal, and geometric quantum and classical correlations are given by Eqs. (15),

- if $c_{i}^{2}<c_{j}^{2}=c_{k}^{2}$ where $i \neq j \neq k$, then only measurements $M$ with $n_{i}^{2}=0$ and $n_{j}^{2}+n_{k}^{2}=1$ are optimal, and geometric quantum and classical correlations are given by Eqs. (15),

- if $c_{i}^{2}<c_{j}^{2}<c_{k}^{2}$ where $i \neq j \neq k$, then only measurements $M$ with $n_{i}^{2}=n_{j}^{2}=0$ and $n_{k}^{2}=1$ are optimal, and geometric quantum and classical correlations are given by Eqs. (15),

where coefficients $w_{1}, w_{2}$ and $w_{3}$ are given by Eqs. (14). The above results show that geometric classical and quantum correlations based on the Bures distance for two-qubit Bell diagonal states are also uniquely determined under the second strategy, despite the fact that for a wide class of two-qubit Bell diagonal states we have more than one optimal measurement $M$ which means that for those states the classical-quantum state $M(\rho)$ cannot be uniquely determined. Therefore, in general non-uniqueness of $M(\rho)$ does not necessarily imply non-uniqueness of geometric quantum correlations under the second strategy. Remarkably, the both strategies provide the same results with regard to the geometric classical and quantum correlations based on the Bures distance and the optimal measurements $M$.

\section{Geometric classical and quantum correlations based on the trace distance}

In this section, we consider geometric classical and quantum correlations based on the trace distance, induced by the Schatten 1-norm, for two-qubit Bell diagonal states to show that the Brodutch and Modi method should be modified as in this case one of two possible strategies results in the non-uniqueness of geometric classical correlations. Moreover, we show how to modify the Brodutch and Modi method to avoid the nonunique results in the general case.

One can apply the Brodutch and Modi method to define geometric quantum and classical correlations present in a multipartite state $\rho$ by adopting the Schatten 1-norm of an operator $\rho-\tau$ as a function $K[\rho, \tau]$

$$
\begin{aligned}
& Q_{T}(\rho)=d_{T}(\rho, M(\rho)), \\
& C_{T}(\rho)=d_{T}\left(M(\rho), M\left(\pi_{\rho}\right)\right),
\end{aligned}
$$

with $d_{T}(\rho, \tau)=\|\rho-\tau\|_{1}$ where $\|X\|_{1}=\operatorname{Tr}\left[\sqrt{X^{\dagger} X}\right]$, and the measurement $M$ is chosen according to one of the above strategies.

It is worth noting here that if $\rho$ is a bipartite state and the measurement $M$ is chosen according to the first strategy, then the geometric quantum correlations (16) coincide with the trace distance geometric quantum discord introduced in [17] if and only if the measured subsystem is a qubit [25]. Although the trace distance geometric quantum discord was evaluated explicitly for two-qubit Bell diagonal states $[17,25,26]$, for 
these states all optimal measurements $M$ and in consequence all classical-quantum states $M(\rho)$ have not yet been identified.

It can be shown that in the case of two-qubit Bell diagonal states and the measurement $M$ described by $\left\{\Pi_{ \pm}\right\}$the Schatten 1-norm of operators $\rho-M(\rho)$ and $M(\rho)-M\left(\pi_{\rho}\right)$ is given by

$$
\begin{aligned}
\|\rho-M(\rho)\|_{1} & =\frac{1}{2}\left(c_{1}^{2}+c_{2}^{2}+c_{3}^{2}-c_{1}^{2} n_{1}^{2}-c_{2}^{2} n_{2}^{2}-c_{3}^{2} n_{3}^{2}\right. \\
& \left.-2\left(c_{2}^{2} c_{3}^{2} n_{1}^{2}+c_{1}^{2} c_{3}^{2} n_{2}^{2}+c_{1}^{2} c_{2}^{2} n_{3}^{2}\right)^{\frac{1}{2}}\right)^{\frac{1}{2}} \\
& +\frac{1}{2}\left(c_{1}^{2}+c_{2}^{2}+c_{3}^{2}-c_{1}^{2} n_{1}^{2}-c_{2}^{2} n_{2}^{2}-c_{3}^{2} n_{3}^{2}\right. \\
& \left.+2\left(c_{2}^{2} c_{3}^{2} n_{1}^{2}+c_{1}^{2} c_{3}^{2} n_{2}^{2}+c_{1}^{2} c_{2}^{2} n_{3}^{2}\right)^{\frac{1}{2}}\right)^{\frac{1}{2}} \\
\left\|M(\rho)-M\left(\pi_{\rho}\right)\right\|_{1} & =\left(c_{1}^{2} n_{1}^{2}+c_{2}^{2} n_{2}^{2}+c_{3}^{2} n_{3}^{2}\right)^{\frac{1}{2}} .
\end{aligned}
$$

The explicit expressions for $\left\|M(\rho)-M\left(\pi_{\rho}\right)\right\|_{1}$ and $\|\rho-M(\rho)\|_{1}$ make it possible to obtain geometric classical and quantum correlations for two-qubit Bell diagonal states under the two natural strategies of choosing the measurement $M$.

\subsection{Strategy 1}

In the framework of the first strategy, for a given two-qubit Bell diagonal state (5) we first identify the measurements $M$ that minimize the geometric quantum correlations (16) and then we use these optimal measurements to compute the geometric classical correlations (17). In other words, for a given point $\left(c_{1}, c_{2}, c_{3}\right)$ of tetrahedron (6) we first identify unit vectors $\left(n_{1}, n_{2}, n_{3}\right)$ that minimize $\|\rho-M(\rho)\|_{1}$ given by Eq. (18) and then we use these optimal vectors to compute $\left\|M(\rho)-M\left(\pi_{\rho}\right)\right\|_{1}$ given by Eq. (19).

It can be shown that under this strategy

- if $c_{i}^{2}=c_{j}^{2}=c_{k}^{2}$ where $i \neq j \neq k$, then all measurements $M$ are optimal, and geometric quantum and classical correlations are given by

$$
\begin{aligned}
& Q_{T}(\rho)=\left|c_{j}\right|, \\
& C_{T}(\rho)=\left|c_{k}\right|,
\end{aligned}
$$

- if $c_{i}^{2}=c_{j}^{2}<c_{k}^{2}$ where $i \neq j \neq k$, then only measurements $M$ with $n_{i}^{2}=n_{j}^{2}=0$ and $n_{k}^{2}=1$ are optimal, and geometric quantum and classical correlations are given by Eqs. (20), 


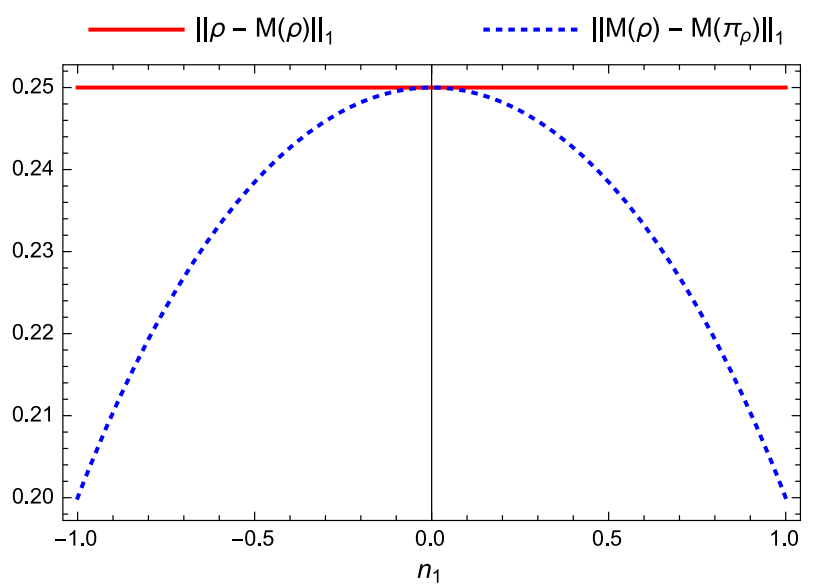

Fig. 1 (Color online) The Schatten 1-norm of operators $\rho-M(\rho)$ and $M(\rho)-M(\pi \rho)$ as a function of $n_{1}$ for two-qubit Bell diagonal state with $c_{1}=1 / 5, c_{2}=1 / 4$ and $c_{3}=1 / 4$ (the third case of the first strategy). For this state, all measurements $M$ are optimal and $\left\|M(\rho)-M\left(\pi_{\rho}\right)\right\|_{1}$ cannot be uniquely determined

- if $c_{i}^{2}<c_{j}^{2}=c_{k}^{2}$ where $i \neq j \neq k$, then all measurements $M$ are optimal, and geometric quantum and classical correlations are given by (see Fig. 1)

$$
\begin{aligned}
& Q_{T}(\rho)=\left|c_{j}\right|, \\
& C_{T}(\rho)=\sqrt{c_{k}^{2}+\left(c_{i}^{2}-c_{k}^{2}\right) n_{i}^{2}},
\end{aligned}
$$

- if $c_{i}^{2}<c_{j}^{2}<c_{k}^{2}$ where $i \neq j \neq k$, then only measurements $M$ with $0 \leq n_{i}^{2} \leq$ $\left(c_{j}^{2}-c_{i}^{2}\right) /\left(c_{k}^{2}-c_{i}^{2}\right), n_{j}^{2}=0$ and $n_{k}^{2}=1-n_{i}^{2}$ are optimal, and geometric quantum and classical correlations are given by (see Fig. 2)

$$
\begin{aligned}
& Q_{T}(\rho)=\left|c_{j}\right|, \\
& C_{T}(\rho)=\sqrt{c_{k}^{2}+\left(c_{i}^{2}-c_{k}^{2}\right) n_{i}^{2}} .
\end{aligned}
$$

The above results show that for a wide class of two-qubit Bell diagonal states we have more than one optimal measurement $M$ which means that for those states the classicalquantum state $M(\rho)$ and in consequence $\left\|M(\rho)-M\left(\pi_{\rho}\right)\right\|_{1}$ cannot be uniquely determined, contrary to recent claims made in $[17,26]$ where for a given Bell diagonal state only one optimal measurement $M$ has been identified. In other words, the above results show explicitly that in the framework of the first strategy of choosing measurement $M$ the non-uniqueness of $M(\rho)$ implies the non-uniqueness of geometric classical correlations based on the trace distance for a wide class of two-qubit Bell diagonal states. The first explicit example of such ambiguities was given in [27] where a different method of constructing meaningful measures of correlations based on their independency was proposed to avoid this problem.

Let us note that the Broduch and Modi method can always result in uniquely determined measures of classical correlations under the first strategy of choosing 


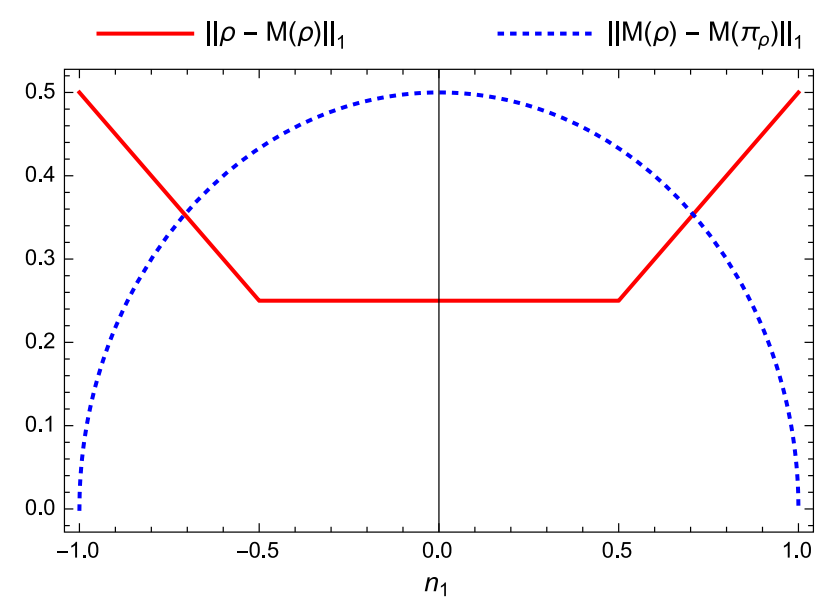

Fig. 2 (Color online) The Schatten 1-norm of operators $\rho-M(\rho)$ and $M(\rho)-M\left(\pi_{\rho}\right)$ as a function of $n_{1}$ for two-qubit Bell diagonal state with $c_{1}=0, c_{2}=1 / 4$ and $c_{3}=1 / 2$ (the fourth case of the first strategy). For this state, only measurements $M$ with $0 \leq n_{1}^{2} \leq 1 / 4, n_{2}^{2}=0$ and $n_{3}^{2}=1-n_{1}^{2}$ are optimal and $\left\|M(\rho)-M\left(\pi_{\rho}\right)\right\|_{1}$ cannot be uniquely determined

measurement $M$, provided that we modify this strategy in the following way. If the classical correlations $C(\rho)$ are not uniquely determined by the minimization procedure, then the classical correlations $C(\rho)$ are additionally maximized over the all measurements $M$ that minimizes the quantum correlations $Q(\rho)$. Interestingly, a similar way of determining classical correlations was proposed in [23] where an alternative approach to geometric classical and quantum correlations based on the Bures distance was considered in the case of two-qubit Bell diagonal states.

It is worth noting here that under the modified version of the first strategy

- if $c_{i}^{2}=c_{j}^{2}=c_{k}^{2}$ where $i \neq j \neq k$, then all measurements $M$ are optimal, and geometric quantum and classical correlations are given by

$$
\begin{aligned}
& Q_{T}(\rho)=\left|c_{j}\right|, \\
& C_{T}(\rho)=\left|c_{k}\right|,
\end{aligned}
$$

- if $c_{i}^{2}=c_{j}^{2}<c_{k}^{2}$ where $i \neq j \neq k$, then only measurements $M$ with $n_{i}^{2}=n_{j}^{2}=0$ and $n_{k}^{2}=1$ are optimal, and geometric quantum and classical correlations are given by Eqs. (23),

- if $c_{i}^{2}<c_{j}^{2}=c_{k}^{2}$ where $i \neq j \neq k$, then only measurements $M$ with $n_{i}^{2}=0$ and $n_{j}^{2}+n_{k}^{2}=1$ are optimal, and geometric quantum and classical correlations are given by Eqs. (23),

- if $c_{i}^{2}<c_{j}^{2}<c_{k}^{2}$ where $i \neq j \neq k$, then only measurements $M$ with $n_{i}^{2}=n_{j}^{2}=0$ and $n_{k}^{2}=1$ are optimal, and geometric quantum and classical correlations are given by Eqs. (23). 


\subsection{Strategy 2}

In the framework of the second strategy, for a given two-qubit Bell diagonal state (5) we first identify the measurements $M$ that maximize the geometric classical correlations (17) and then we use these optimal measurements to compute the geometric quantum correlations (16). In other words, for a given point $\left(c_{1}, c_{2}, c_{3}\right)$ of tetrahedron (6) we first identify unit vectors $\left(n_{1}, n_{2}, n_{3}\right)$ that maximize $\left\|M(\rho)-M\left(\pi_{\rho}\right)\right\|_{1}$ given by Eq. (19) and then we use these optimal vectors to compute $\|\rho-M(\rho)\|_{1}$ given by Eq. (18).

It can be shown that under this strategy

- if $c_{i}^{2}=c_{j}^{2}=c_{k}^{2}$ where $i \neq j \neq k$, then all measurements $M$ are optimal, and geometric quantum and classical correlations are given by

$$
\begin{aligned}
& Q_{T}(\rho)=\left|c_{j}\right|, \\
& C_{T}(\rho)=\left|c_{k}\right|,
\end{aligned}
$$

- if $c_{i}^{2}=c_{j}^{2}<c_{k}^{2}$ where $i \neq j \neq k$, then only measurements $M$ with $n_{i}^{2}=n_{j}^{2}=0$ and $n_{k}^{2}=1$ are optimal, and geometric quantum and classical correlations are given by Eqs. (24),

- if $c_{i}^{2}<c_{j}^{2}=c_{k}^{2}$ where $i \neq j \neq k$, then only measurements $M$ with $n_{i}^{2}=0$ and $n_{j}^{2}+n_{k}^{2}=1$ are optimal, and geometric quantum and classical correlations are given by Eqs. (24),

- if $c_{i}^{2}<c_{j}^{2}<c_{k}^{2}$ where $i \neq j \neq k$, then only measurements $M$ with $n_{i}^{2}=n_{j}^{2}=0$ and $n_{k}^{2}=1$ are optimal, and geometric quantum and classical correlations are given by Eqs. (24).

The above results show that both $\left\|M(\rho)-M\left(\pi_{\rho}\right)\right\|_{1}$ and $\|\rho-M(\rho)\|_{1}$ are uniquely determined, despite the fact that for a wide class of two-qubit Bell diagonal states we have more than one optimal measurement $M$. Moreover, it is worth noting here that the above expressions for classical and geometric quantum correlations based on the trace distance remarkably coincide with those one can obtain in the framework of the modified version of the first strategy of choosing measurement $M$.

The question whether the Brodutch and Modi method always results in uniquely determined measures of quantum correlations under the second strategy of choosing measurement $M$ remains open for the future research. However, if the answer will be negative, then the second strategy of choosing measurement $M$ should be modified in the following way. If the quantum correlations $Q(\rho)$ are not uniquely determined by the maximization procedure, then the quantum correlations $Q(\rho)$ are additionally minimized over the all measurements $M$ that maximizes the classical correlations $C(\rho)$.

\section{Conclusions}

In the framework of the Brodutch and Modi method, quantum correlations are quantified by a distance between a given multipartite state $\rho$ and the classical-quantum state 
$M(\rho)$ emerging from a measurement $M$ performed on the considered state. However, classical correlations are quantified by a distance between the post-measurement classical-quantum state $M(\rho)$ and the completely separable state $M\left(\pi_{\rho}\right)$ resulting from the same measurement performed on the tensor product of the states of the individual subsystems. Within this method, there are two natural strategies of choosing a measurement $M$ that provide meaningful measures of classical and quantum correlations present in a multipartite state $\rho$. In both strategies, $M$ is a non-selective projective measurement performed on one subsystem of the multipartite system in a state $\rho$. However, the measurement $M$ minimizes quantum correlations according to the first strategy, and it maximizes classical correlations according to the second one.

In this work, we have applied the Brodutch and Modi method to obtain for the first time geometric classical and quantum correlations based on the Bures distance under the two natural strategies of choosing the measurement $M$ performed on the first qubit of the two-qubit system in a Bell diagonal state. Under the first strategy, we first identified the measurements $M$ that minimize the geometric quantum correlations and then we used these optimal measurements to compute the geometric classical correlations, while under the second one, we first identified the measurements $M$ that maximize the geometric classical correlations and then we used these optimal measurements to compute the geometric quantum correlations. For the both strategies, we have identified not only all optimal measurements $M$ but also all two-qubit Bell diagonal states for which there exists more than one optimal measurement $M$. However, the nonuniqueness of $M(\rho)$ does not affect the geometric classical and quantum correlations for the first and the second strategy, respectively. Remarkably, it turned out that the both strategies provide the same results with regard to the geometric classical and quantum correlations and the optimal measurements.

Moreover, we have shown that the Brodutch and Modi method should be modified as in general it may provide non-unique results. As an explicit example, we have applied the Brodutch and Modi method to obtain geometric classical and quantum correlations based on the trace distance under the two natural strategies of choosing the measurement $M$ performed on the first qubit of the two-qubit system in a Bell diagonal state. For the both strategies, we have computed the geometric classical and quantum correlations, although the geometric quantum correlations under the first strategy had been studied in the literature. Moreover, for the both strategies, we have identified not only all optimal measurements $M$ but also all two-qubit Bell diagonal states for which there exists more than one optimal measurement $M$. Remarkably, it turned out that for the first strategy the non-uniqueness of $M(\rho)$ results in the nonuniqueness of geometric classical correlations, and therefore, the Brodutch and Modi method should be modified. Finally, we have shown how to modify the Brodutch and Modi method to avoid the problem of non-unique results with regard to classical and quantum correlations in the general case.

Acknowledgements We thank Paweł Caban for helpful comments. This work was supported by the University of Lodz. Iwona Wintrowicz acknowledges the support from the European Union under the Human Capital Operational Programme, Measure 8.2.1.

Open Access This article is distributed under the terms of the Creative Commons Attribution 4.0 International License (http://creativecommons.org/licenses/by/4.0/), which permits unrestricted use, distribution, 
and reproduction in any medium, provided you give appropriate credit to the original author(s) and the source, provide a link to the Creative Commons license, and indicate if changes were made.

\section{References}

1. Horodecki, R., Horodecki, P., Horodecki, M., Horodecki, K.: Quantum entanglement. Rev. Mod. Phys. 81, 865 (2009)

2. Modi, K., Brodutch, A., Cable, H., Paterek, T., Vedral, V.: The classical-quantum boundary for correlations: discord and related measures. Rev. Mod. Phys. 84, 1655 (2012)

3. Werner, R.F.: Quantum states with Einstein-Podolsky-Rosen correlations admitting a hidden-variable model. Phys. Rev. A 40, 4277 (1989)

4. Knill, E., Laflamme, R.: Power of one bit of quantum information. Phys. Rev. Lett. 81, 5672 (1998)

5. Braunstein, S.L., Caves, C.M., Jozsa, R., Linden, N., Popescu, S., Schack, R.: Separability of very noisy mixed states and implications for NMR quantum computing. Phys. Rev. Lett. 83, 1054 (1999)

6. Bennett, C.H., DiVincenzo, D.P., Fuchs, C.A., Mor, T., Rains, E., Shor, P.W., Smolin, J.A., Wootters, W.K.: Quantum nonlocality without entanglement. Phys. Rev. A 59, 1070 (1999)

7. Meyer, D.A.: Sophisticated quantum search without entanglement. Phys. Rev. Lett. 85, 2014 (2000)

8. Biham, E., Brassard, G., Kenigsberg, D., Mor, T.: Quantum computing without entanglement. Theor. Comput. Sci. 320, 15 (2004)

9. Datta, A., Flammia, S.T., Caves, C.M.: Entanglement and the power of one qubit. Phys. Rev. A 72, 042316 (2005)

10. Datta, A., Vidal, G.: Role of entanglement and correlations in mixed-state quantum computation. Phys. Rev. A 75, 042310 (2007)

11. Ollivier, H., Zurek, W.H.: Quantum discord: a measure of the quantumness of correlations. Phys. Rev. Lett. 88, 017901 (2001)

12. Henderson, L., Vedral, V.: Classical, quantum and total correlations. J. Phys. A 34, 6899 (2001)

13. Datta, A., Shaji, A., Caves, C.M.: Quantum discord and the power of one qubit. Phys. Rev. Lett. 100, 050502 (2008)

14. Modi, K., Paterek, T., Son, W., Vedral, V., Williamson, M.: Unified view of quantum and classical correlations. Phys. Rev. Lett. 104, 080501 (2010)

15. Dakić, B., Vedral, V., Brukner, Č.: Necessary and sufficient condition for nonzero quantum discord. Phys. Rev. Lett. 105, 190502 (2010)

16. Piani, M.: Problem with geometric discord. Phys. Rev. A 86, 034101 (2012)

17. Paula, F.M., de Oliveira, T.R., Sarandy, M.S.: Geometric quantum discord through the Schatten 1-norm. Phys. Rev. A 87, 064101 (2013)

18. Debarba, T., Maciel, T.O., Vianna, R.O.: Witnessed entanglement and the geometric measure of quantum discord. Phys. Rev. A 86, 024302 (2012)

19. Brodutch, A., Modi, K.: Criteria for measures of quantum correlations. Quantum Inf. Comput. 12, 0721 (2012)

20. Horodecki, R., Horodecki, M.: Information-theoretic aspects of inseparability of mixed states. Phys. Rev. A 54, 1838 (1996)

21. Aaronson, B., Lo Franco, R., Adesso, G.: Comparative investigation of the freezing phenomena for quantum correlations under nondissipative decoherence. Phys. Rev. A 88, 012120 (2013)

22. Spehner, D., Orszag, M.: Geometric quantum discord with Bures distance: the qubit case. J. Phys. A 47, 035302 (2014)

23. Bromley, T.R., Cianciaruso, M., Lo Franco, R., Adesso, G.: Unifying approach to the quantification of bipartite correlations by Bures distance. J. Phys. A 47, 405302 (2014)

24. Roga, W., Spehner, D., Illuminati, F.: Geometric measures of quantum correlations: characterization, quantification, and comparison by distances and operations. J. Phys. A 49, 235301 (2016)

25. Nakano, T., Piani, M., Adesso, G.: Negativity of quantumness and its interpretations. Phys. Rev. A 88, 012117 (2013)

26. Paula, F.M., Montealegre, J.D., Saguia, A., de Oliveira, T.R., Sarandy, M.S.: Geometric classical and total correlations via trace distance. Europhys. Lett. 103, 50008 (2013)

27. Paula, F.M., Saguia, A., de Oliveira, T.R., Sarandy, M.S.: Overcoming ambiguities in classical and quantum correlation measures. Europhys. Lett. 108, 10003 (2014) 